\title{
An unusual pulmonary condition presenting following trauma
}

\author{
John RA Turkington FRCR ${ }^{1}$, Eamon MCAteer FRCR ${ }^{1}$, Rory P Convery FRCP ${ }^{2}$
}

\begin{abstract}
JRA Turkington, E McAteer, RP Convery. An unusual pulmonary condition presenting following trauma. Can Respir J 2006;13(7):384-386.
\end{abstract}

An 18-year-old man presented to the emergency department following an assault. He complained of left-sided pleuritic chest pain and difficulty breathing. Clinical examination revealed reduced air entry and coarse crepitations at the left lung base. A chest $\mathrm{x}$-ray showed a large opacity at the left lung base that contained multiple cystic areas with air-fluid levels. Due to the history of trauma, a provisional diagnosis of a ruptured hemidiaphragm with small bowel herniation was made. Further imaging, including ultrasound, spiral computed tomography and magnetic resonance angiography, showed an aberrant vessel supplying the opacity and drainage into the pulmonary venous system. A diagnosis of a bronchopulmonary sequestration (intralobar type) was made. The differential diagnosis of the radiographic appearance is also discussed.

Key Words: Bronchopulmonary sequestration; Magnetic resonance angiography; Tomography, spiral computed; Ultrasonography

\section{Un problème pulmonaire inhabituel après un traumatisme}

\begin{abstract}
Un homme de 18 ans s'est présenté à l'urgence après des voies de fait. Il souffrait de douleurs thoraciques pleurétiques gauches et de troubles respiratoires. L'examen clinique a révélé une entrée d'air limitée et des crépitations grossières à la base du poumon gauche. Une radiographie pulmonaire a démontré une grande opacité à la base du poumon gauche, contenant de nombreuses zones kystiques avec niveau hydroaérique. Étant donné la nature du traumatisme, on a posé un diagnostic provisoire de rupture de l'hémidiaphragme avec herniation de l'intestin grêle. Une imagerie plus complexe, y compris une échographie, une tomodensitométrie hélicoïdale et une angiographie par résonance magnétique, a révélé la présence d'un vaisseau aberrant alimentant l'opacité et un drainage dans le système veineux pulmonaire. On a alors posé un diagnostic de séquestration bronchopulmonaire (de type intralobaire). Le diagnostic différentiel de l'aspect radiographique est également abordé.
\end{abstract}

\section{CASE PRESENTATION}

An 18-year-old man presented to the emergency department six days following an assault in which he had been knocked to the ground. He complained of difficulty breathing and also of left-sided pleuritic chest pain.

The patient had no past medical history of note; specifically, he had no history of previous respiratory problems or recurrent chest infections. However, he did give a history of a sports injury in the past, in which he had received an abdominal blow that severely 'winded' him.

On admission, the patient was afebrile, and an ear, nose and throat examination revealed a nasal septal hematoma. Air entry was reduced at the left lung base, and coarse basal crepitations were heard on auscultation. The remainder of the clinical examination was unremarkable. The patient's white blood cell count and hemoglobin concentration were within normal limits.

The nasal septal hematoma was drained by an ear, nose and throat surgeon, and a chest $\mathrm{x}$-ray (CXR) was performed. The CXR (Figure 1) showed a large abnormal opacity at the left lung base, which contained multiple similarly sized, rounded lucent areas. In the upper abdomen, gas was present within a normal-calibre, large bowel, with a paucity of small bowel gas below the left hemidiaphragm.
Due to the history of trauma, left-sided chest pain and the CXR appearance, a provisional clinical diagnosis of a ruptured left hemidiaphragm, with herniation of loops of small bowel into the chest, was made.

A computed tomography (CT) scan of the chest and abdomen showed a large, left-sided, basal pulmonary mass containing multiple cysts, many containing air-fluid levels (Figure 2). The intra-abdominal organs, including the small and large bowel, were normally situated below the diaphragm. A contrast-enhanced vessel arising from the descending thoracic aorta was found to be supplying the mass. Contrary to the provisional diagnosis, the cystic mass was not a ruptured hemidiaphragm with small bowel herniation.

A magnetic resonance (MR) scan with MR angiography proved to be the definitive test. Again, a large left-sided, basal intrapulmonary multicystic mass was seen. The cysts were of varying signal intensities on $\mathrm{T} 2$-weighted images, with some cysts containing fluid (high signal intensity) and others of lower signal intensity (probably representing mucinous fluid), and others containing air (signal void). A small left pleural effusion was also present. The MR angiogram clearly showed an abnormal vessel arising from the descending thoracic aorta, supplying the mass, and venous drainage to the left inferior pulmonary vein was also observed (Figures 3 and 4).

${ }^{1}$ Departments of Radiology and ${ }^{2}$ Respiratory Medicine, Craigavon Area Hospital, Craigavon, Northern Ireland, United Kingdom

Correspondence: Dr John RA Turkington, 9 Farm Lodge Way, Greenisland BT38 8YA, Northern Ireland, United Kingdom.

Telephone 011-44-28-3833-4444, fax 011-44-28-3835-0068, e-mail jraturkington@hotmail.com 


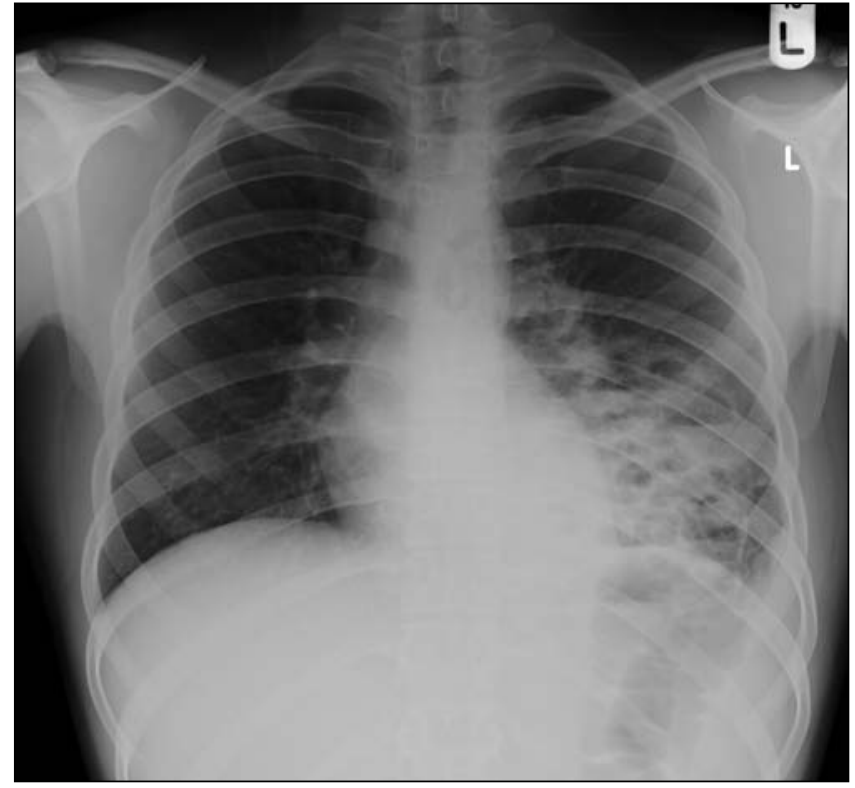

Figure 1) Chest $x$-ray showing a soft-tissue density opacity at the left lung base containing multiple cystic areas

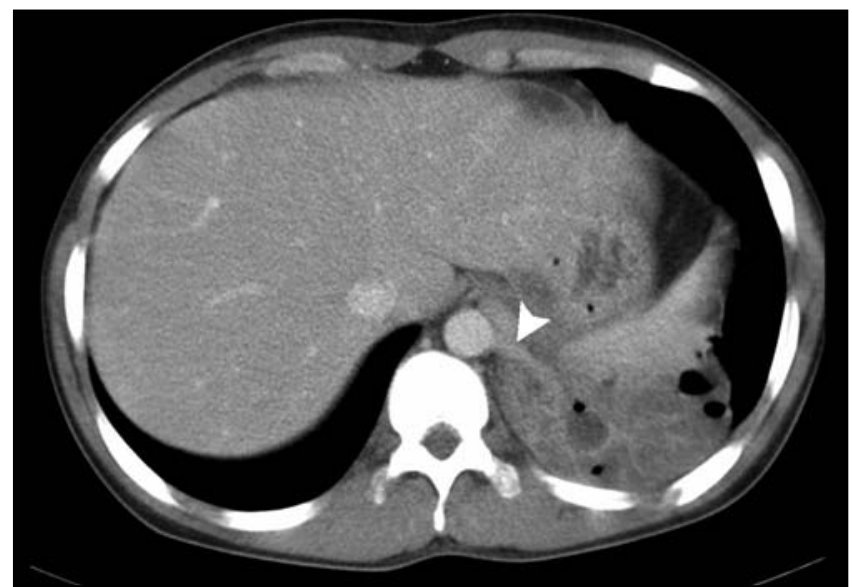

Figure 2) Contrast-enhanced computed tomography showing the left intrapulmonary soft-tissue mass containing multiple air-fluid levels. A vessel (arrowhead) can be identified arising from the descending thoracic aorta and entering the mass

A diagnosis of intralobar bronchopulmonary sequestration was made.

\section{DISCUSSION}

Bronchopulmonary sequestration is an uncommon congenital pulmonary malformation consisting of a nonfunctioning segment of lung that does not communicate with the bronchial tree and receives a systemic rather than a pulmonary arterial blood supply (1). These malformations can be classified into two types (intralobar or extralobar), depending on their pleural covering and their venous drainage.

Extralobar bronchopulmonary sequestration is the least common of the two types, accounting for $14 \%$ to $25 \%$ of cases. It has its own visceral pleural covering and usually manifests in neonates, with $60 \%$ presenting within the first six months. It occurs more commonly on the left side, particularly between the diaphragm and the left lower lobe, and is associated with

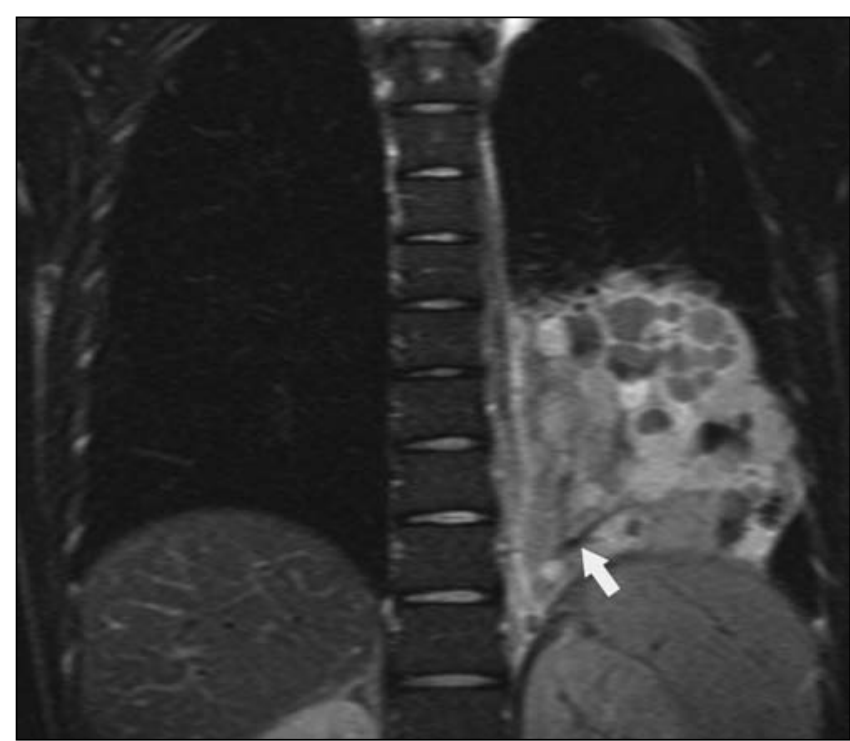

Figure 3) Coronal T2-weighted magnetic resonance imaging confirming the aberrant arterial supply (arrow). The multicystic nature of the mass is clearly seen, with the cysts varying in signal intensity, because some contain fluid and others probably contain mucous. A small left pleural effusion is present

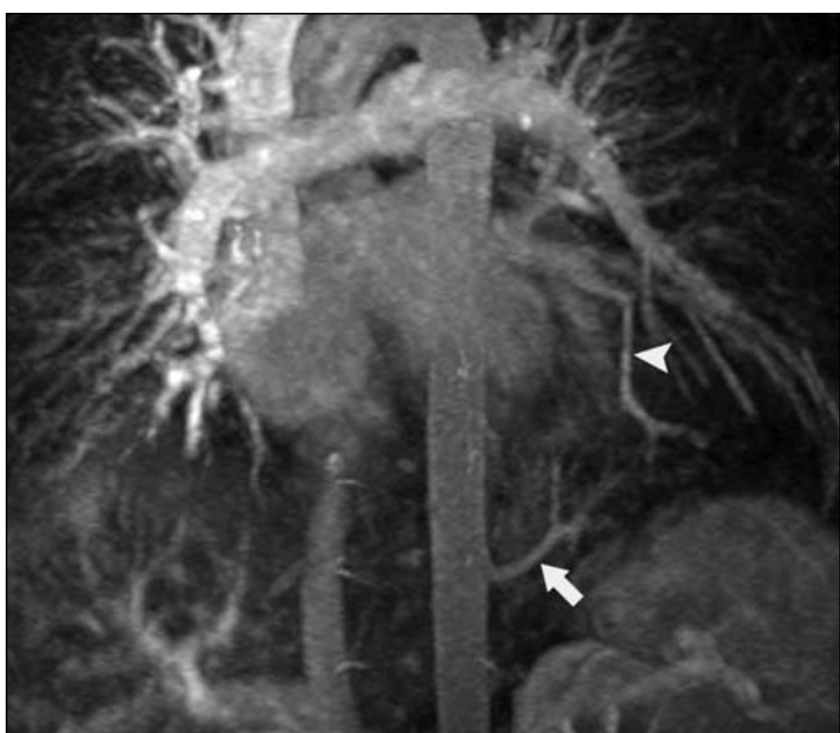

Figure 4) Contrast-enhanced magnetic resonance coronal angiogram image confirming the aberrant systemic arterial supply arising from the descending thoracic aorta (arrow). The draining vein can be seen draining into the left inferior pulmonary vein (arrowhead)

other congenital abnormalities, including cardiac, diaphragmatic and pulmonary abnormalities, in $15 \%$ to $65 \%$ of cases (2). It has a systemic arterial blood supply usually arising from the aorta, and it drains via systemic veins, most commonly via the inferior vena cava, azygous or hemiazygous veins.

In contrast, intralobar bronchopulmonary sequestration (as in the present case) is more common, accounting for $75 \%$ to $86 \%$ of cases $(1,2)$. It is covered by the visceral pleura of the affected pulmonary lobe and does not communicate with the bronchial tree. Most cases present in adults and are rarely associated with other congenital abnormalities (3). The posterior basal segment of the lung is more often affected than the upper lobe, and the majority 
of cases (66\%) occur on the left (4). The CXR appearance of an intralobar bronchopulmonary sequestration is typically that of a pulmonary mass containing cysts, cavitations and possibly fluid levels (3). The cavitations are caused by aeration of the sequestered segment due to alveolar drift from normal lung via the pores of Kohn. The arterial supply is usually via a single largediameter vessel arising from the distal thoracic aorta (75\%) (5). This vessel can also arise from the proximal abdominal aorta, celiac axis or splenic artery (22\%) (5). Venous drainage is to the left atrium via the pulmonary veins.

Traditionally, conventional angiography has been the gold standard for the diagnostic demonstration of the aberrant arterial supply. More recently, however, noninvasive imaging techniques are proving useful in the demonstration of the aberrant vessels, particularly those that do not involve ionizing radiation, such as MR angiography.

Ultrasound can show an echogenic solid mass or cystic/solid mass, and duplex scanning of vessels within the mass can assess its arterial blood supply and its venous drainage (6). When using ultrasound, it can be difficult to identify whether the arterial supply arises from the aorta and, if the aberrant vessel is small, it may not be identified (7).

The CT appearance of bronchopulmonary sequestration is usually that of a homogenous or inhomogenous solid mass with or without cystic changes or fluid levels. Surrounding lung parenchymal emphysematous changes are characteristic and are caused by collateral air drift (4). The likelihood of visualizing the aberrant vessel is increased with helical and multidetector CT scanning with multiplanar reconstructions (8). The venous drainage can be difficult to clearly identify with CT.

MR imaging is extremely useful because it does not involve ionizing radiation and, combined with MR angiography, can precisely identify the supplying aberrant arterial supply as well as the venous drainage $(4,9)$. Determining the anatomical origin of these vessels, which may be multiple, is important to prevent intraoperative hemorrhage during resection of the sequestration. In the present case of venous drainage to the pulmonary venous system, the sequestration was of the intralobar type.

Most patients with an intralobar bronchopulmonary sequestration present with recurrent or persistent chest infections, but they may also be asymptomatic $(4,10)$. Pulmonary sequestration has not been previously reported with a traumatic presentation, as in the present case, with the sequestered segment mimicking a ruptured diaphragm. Our patient was only diagnosed after a traumatic incident and because he complained of chest pain and the CXR showed a large multicystic abnormality with air-fluid levels at the left lung base. The differential diagnosis includes pneumonia, pulmonary abscesses, cystic bronchiectasis and cystic adenomatoid malformations (in children), which may have a similar appearance on CXR. Therefore, it is important to further evaluate this type of CXR appearance with cross-sectional imaging.

\section{CONCLUSIONS}

The present case highlights that unusual pulmonary pathologies can present to the emergency department following coincidental trauma.

It is important to be aware that in patients with a recent history of trauma, the presence of an abnormal area of aeration in the chest is not always due to bowel herniation secondary to diaphragmatic rupture. Further investigation with CT scanning should be performed in such cases and can confirm or exclude bowel herniation into the chest. CT can also demonstrate other pathologies that mimic a similar CXR appearance, as discussed above, including bronchopulmonary sequestration, as in the present case. In cases of bronchopulmonary sequestration, noninvasive imaging such as CT, Doppler ultrasound and MR imaging can identify an aberrant arterial blood supply to the sequestered segment, which is diagnostic. MR imaging is now the most reliable noninvasive imaging technique to establish a diagnosis.

\section{REFERENCES}

1. Veerappan GR, Lettieri CJ. Bronchopulmonary sequestration. J Postgrad Med 2003;49:280-1.

2. Torreggiani WC, Logan PM, McElvaney NG. Persistant right lower lobe consolidation. Chest 2000;117:588-90.

3. Lewis MM, Tsou E. A 66-year-old man with dyspnea, left lower lobe infiltrate, and abnormal imaging. Chest 2000;117:1782-6.

4. Au VW, Chan JK, Chan FL. Pulmonary sequestration diagnosed by contrast enhanced three-dimensional MR angiography. Br J Radiol 1999;72:709-11.

5. Lehnhardt S, Winterer JT, Uhrmeister P, Herget G, Laubenberger J. Pulmonary sequestration: Demonstration of blood supply with 2D and 3D MR angiography. Eur J Radiol 2002;44:28-32.

6. Yuan A, Yang PC, Chang DB, Yu CJ, Kuo SH, Luh KT. Lung sequestration. Diagnosis with ultrasound and triplex Doppler technique in an adult. Chest 1992;102:1880-2.

7. Ko SF, Ng SH, Lee TY, et al. Noninvasive imaging of bronchopulmonary sequestration. AJR Am J Roentgenol 2000; $175: 1005-12$.

8. Ahmed M, Jacobi V, Vogl TJ. Multislice CT and CT angiography for non-invasive evaluation of bronchopulmonary sequestration. Eur Radiol 2004;14:2141-3.

9. Zhang M, Zhu J, Wang Q, Shang D. Contrast enhanced MR angiography in pulmonary sequestration. Chin Med J (Engl) 2001;114:1326-8.

10. Zylak CJ, Eyler WR, Spizarny DL, Stone CH. Developmental lung anomalies in the adult: Radiologic-pathologic correlation. Radiographics 2002;22:S25-S43. 


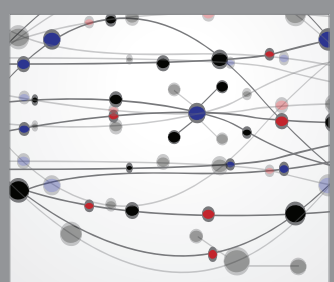

The Scientific World Journal
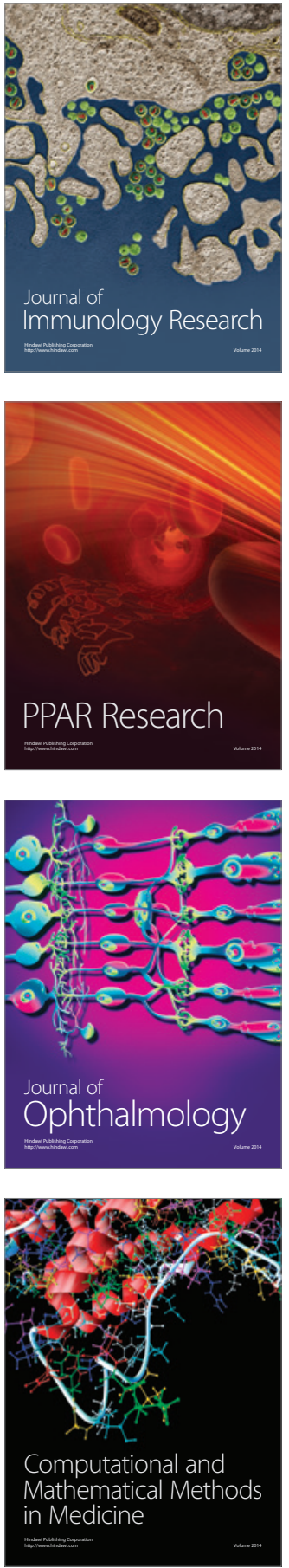

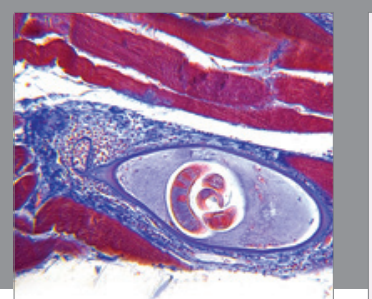

Gastroenterology Research and Practice

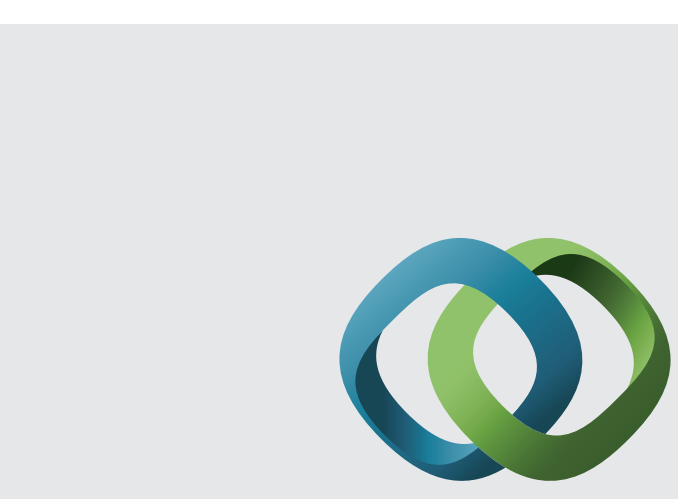

\section{Hindawi}

Submit your manuscripts at

http://www.hindawi.com
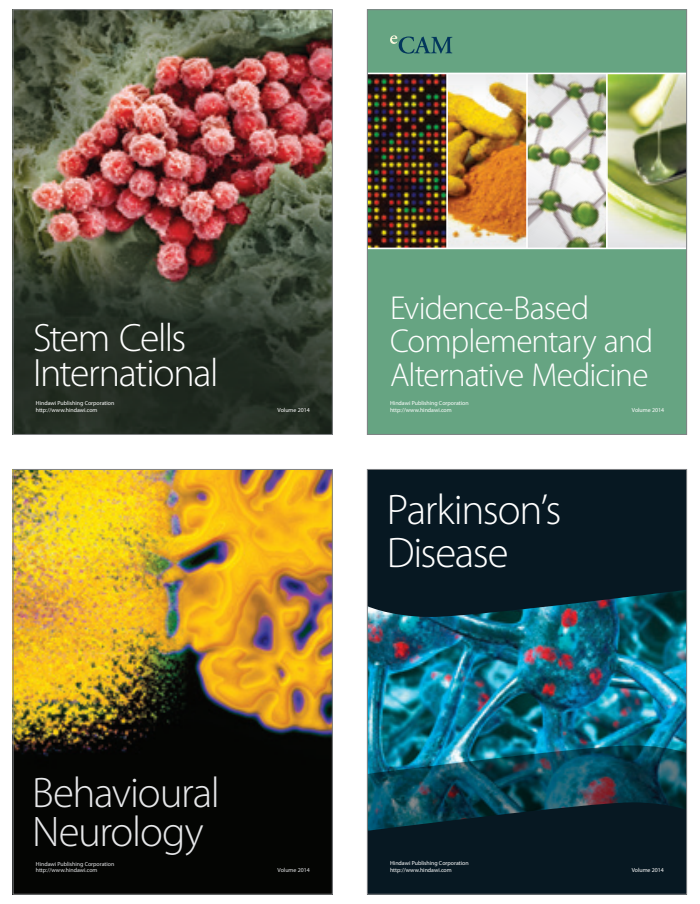
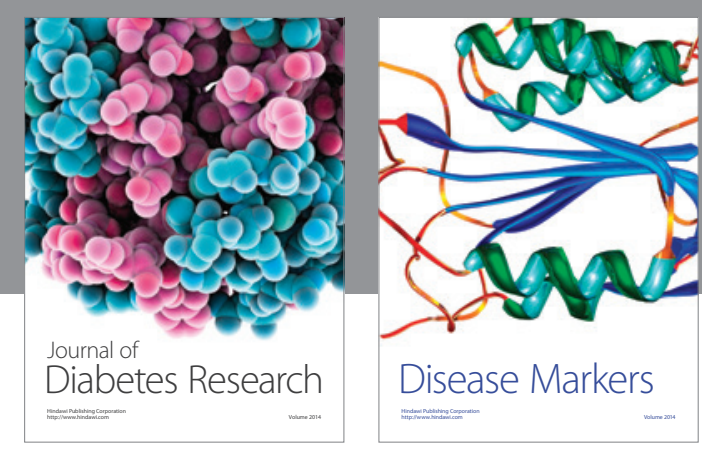

Disease Markers
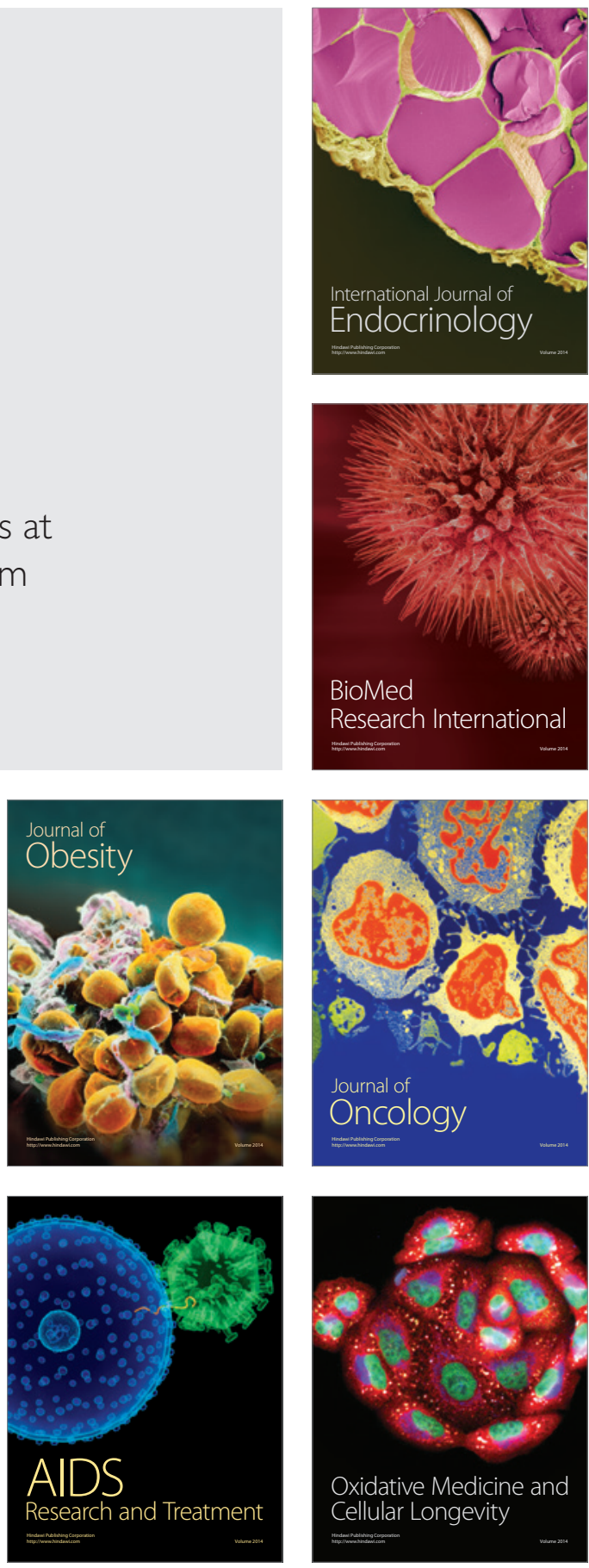